\title{
Physicochemical, electrical and optical studies of methyl-3-(2-furylmethylidene) carbazate single crystal
}

\author{
G GOMATHI and R GOPALAKRISHNAN* \\ Crystal Research Lab, Department of Physics, Anna University, Chennai 600 025, India
}

MS received 5 September 2014; accepted 6 April 2015

\begin{abstract}
The current study provides an insight into the physicochemical properties of an organic single crystal methyl-3-(2-furylmethylidene) carbazate, which was grown by employing the slow evaporation solution growth technique and its results were correlated for application point of view. The grown crystal was confirmed by performing single-crystal $X$-ray diffraction studies and Fourier transform infrared analysis. The optical, thermal, dielectric and mechanical properties of the grown single crystal were primarily investigated. Etching study was performed to analyse the defects and growth mechanism. Kurtz-Perry powder technique was used to study the second harmonic generation efficiency of the crystal and the crystal was found to exhibit Type-I phase matching.
\end{abstract}

Keywords. Organic compound; single crystal growth; characterization; nonlinear optical material.

\section{Introduction}

Nonlinear optical (NLO) organic crystals have proved to be the most interesting candidates for many technological applications, such as second harmonic generation (SHG), frequency mixing, electro-optic modulation, optical parametric oscillation, optical bistability and hybrid photonic integrated circuitry. ${ }^{1,2}$ Furthermore, organic NLO materials are well suited for high-speed and highcapacity optical communication networks such as signal regeneration, time division demultiplexing, logic gating, switching, etc., Exceptionally large intrinsic optical nonlinearities have initiated the research interest towards organic single crystals. It is well demonstrated that the intrinsic nonlinearities are exhibited with higher magnitude in organic single crystals than inorganic dielectric and semiconductor crystals. ${ }^{1}$ NLO effects of organic molecular crystals depend mainly on the polarizability of the electrons in the $\pi$-bonding orbitals, which is in contrast to inorganic materials where lattice vibrations play a dominant role. The fast growing development of optical fibre communication system has also stimulated the search for new NLO materials that are capable of performing fast and efficient processing of optical signal. ${ }^{1}$

In order to deepen our understanding of the fundamental material properties and device physics of organic materials, more attention is to be given to the studies of the physical properties of organic single crystals. ${ }^{5}$ Hence we

*Author for correspondence (krgkrishnan@annauniv.edu, krgkrishnan@yahoo.com) attempted to study the physico-chemical, electrical and optical properties of the compound methyl-3-(2furylmethylidene) carbazate (MFMC), which is an organic compound with the molecular formula $\mathrm{C}_{7} \mathrm{H}_{8} \mathrm{~N}_{2} \mathrm{O}_{3}$. The asymmetric unit of the molecule contains two polar molecules and the molecules are linked into chains by the way of alternating $\mathrm{N}-\mathrm{H} \cdots \mathrm{O}$ and $\mathrm{N}-\mathrm{H} \cdots \mathrm{N}$ (or) $\mathrm{N}-\mathrm{H} \cdots \mathrm{O}$ hydrogen bond linkages. ${ }^{6} \mathrm{Li}^{6}$ has already reported the structural studies of the title compound.

\section{Materials and methods}

As organic crystals have low melting temperatures with high vapour pressures and are soluble in numerous organic solvents, both solution and gas phase methods can be used for crystal growth. ${ }^{5}$ MFMC was synthesized by following the procedure given by $\mathrm{Li}^{6}$ The chemical reaction scheme is shown in scheme 1.

Methyl carbazate (Sigma Aldrich) and furfuraldehyde were taken in $0.1 \mathrm{~mol}$ and refluxed with $20 \mathrm{ml}$ of ethanol for $4 \mathrm{~h}$ to get the title compound. The obtained compound was recrystallized using ethanol as solvent at room temperature and the supersaturated solution was kept for slow evaporation. A small single crystal was chosen as seed crystal and it was allowed to grow in a supersaturated solution of MFMC. A light brown coloured transparent MFMC single crystal of size $1.6 \times 0.5 \times 0.4 \mathrm{~cm}^{3}$ (figure 1a) was harvested from the mother solution after a period of 15 days. The morphology of MFMC single crystal was predicted using WinXMorph software version $1.24,{ }^{7,8}$ which is shown in figure $1 \mathrm{~b}$. 


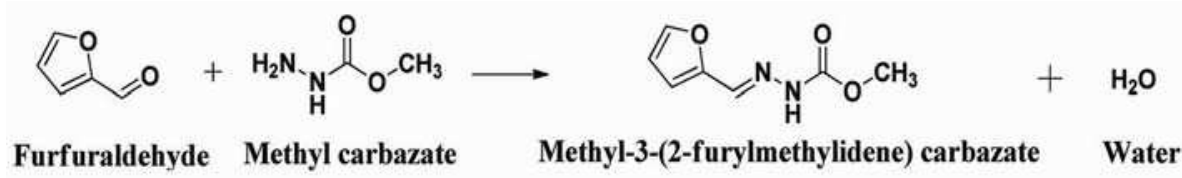

Scheme 1. Synthesis of methyl-3-(2-furylmethylidene) carbazate compound.
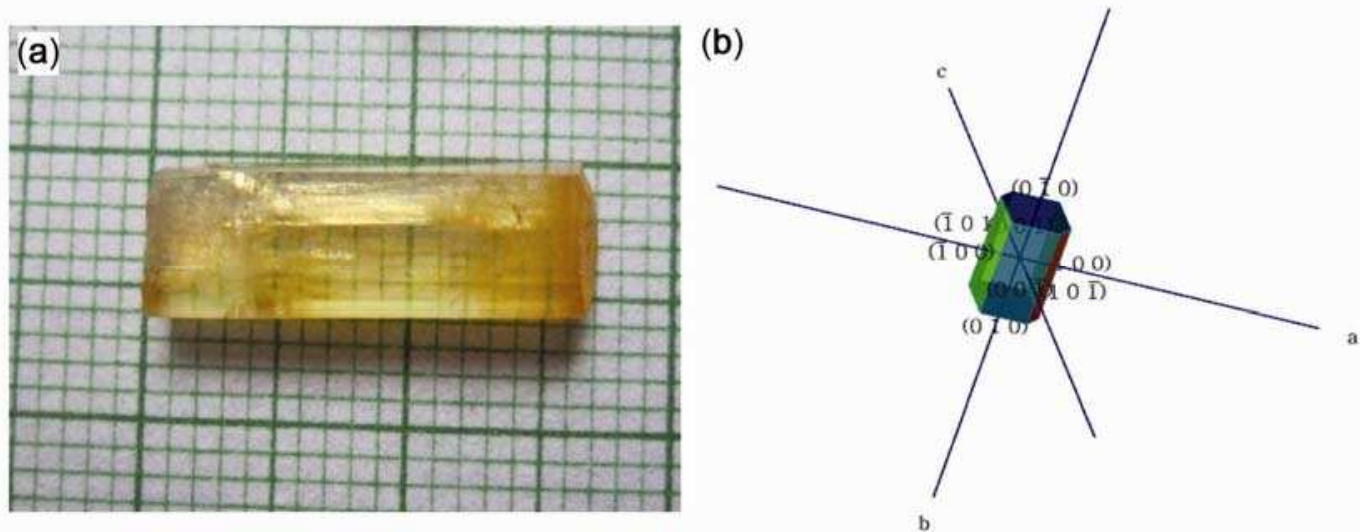

Figure 1. (a) As-grown MFMC single crystal from seed and (b) predicted morphology of MFMC single crystal.

\subsection{Characterization studies}

The single-crystal X-ray diffraction (XRD) analysis of the title compound was carried out using Bruker Nonius X-ray diffractometer, which employs monochromated $\operatorname{MoK} \alpha(\lambda \sim 0.71 \AA)$ radiation. The Fourier transform infrared (FTIR) analysis was carried out using Bruker Alpha FTIR spectrometer in the wavenumber range 400 $4000 \mathrm{~cm}^{-1}$. The UV-visible-diffused reflectance spectrum of the title compound was recorded using Shimadzu $2450 \mathrm{UV}$-vis spectrophotometer in the wavelength range 200-800 $\mathrm{nm}$. The TG-DSC analysis of the title compound with the initial sample weight of $6.7380 \mathrm{mg}$ was carried out between 25 and $500^{\circ} \mathrm{C}$ in nitrogen atmosphere using SDT-Q600 Simultaneous TGA/DSC instrument. The conventional melting point apparatus VEEGO MODEL: VMP-PM was also used to check the melting point of the material. The dielectric measurement of the title compound was done using HIOKI 3532-50 LCR Hitester with the electrode diameter $0.014515 \mathrm{~m}$. The microhardness study of the MFMC crystal was carried out using MATSUZAWA Vickers Microhardness Tester. Optical microscope in the reflection mode of magnification of about $400 \times$ was used to carry out etching studies. In the Kurtz-Perry technique, the frequency-doubled, Q-switched Nd:YAG (Spectra-Physics, INDI 40) laser beam, delivering $6 \mathrm{~ns}$ laser pulses at $1064 \mathrm{~nm}$ at a repetition rate of $10 \mathrm{~Hz}$ (typically Nd: YAG beam at $1064 \mathrm{~nm}$ ) is directed onto powdered samples. The emitted light is collected using a parabolic mirror, filtered to remove the fundamental beam and measured with a suitable detection system (usually consisting of a PMT, lock in amplifier). Urea or KDP is used for calibrating the SHG intensity. The precision of SHG intensity is typically $\pm 10-15 \% .{ }^{9}$

\section{Results and discussion}

\subsection{Single-crystal XRD analysis}

The obtained single-crystal XRD data were compared with those existing in literature ${ }^{6}$ and the data are presented in table 1. It is found that the MFMC crystallizes in monoclinic system with space group C2.

\subsection{FTIR spectral analysis}

The FTIR studies were performed to investigate the presence of functional groups and their vibrational modes and the recorded spectrum as shown in figure 2 . The spectrum confirms the formation of $\mathrm{C}_{7} \mathrm{H}_{8} \mathrm{~N}_{2} \mathrm{O}_{3}$ and its group frequencies and the corresponding assignments are presented in table $2 .^{10,11}$

\subsection{Ultra violet-visible-diffused reflectance spectroscopy $(U V-v i s-D R S)$ analysis}

The UV-vis-DRS spectrum is shown in figure 3 along with the absorbance plot in the inset graph. The maximum 
Table 1. Comparison of single-crystal XRD data.

\begin{tabular}{lll}
\hline Crystal parameters & Present work & Reported values [5] \\
\hline Crystal system, space group & Monoclinic, C2 & Monoclinic, C2 \\
$a(\AA)$ & $14.74(9)$ & $14.668(5)$ \\
$b(\AA)$ & $7.73(4)$ & $7.7356(15)$ \\
$c(\AA)$ & $14.93(9)$ & $14.720(3)$ \\
$\alpha(\mathrm{deg})$ & 90 & 90 \\
$\beta(\mathrm{deg})$ & $105.30(4)$ & $104.11(4)$ \\
$\gamma(\mathrm{deg})$ & 90 & 90 \\
Volume $\left(\AA^{3}\right)$ & $1640(15)$ & $1619.8(7)$ \\
\hline
\end{tabular}

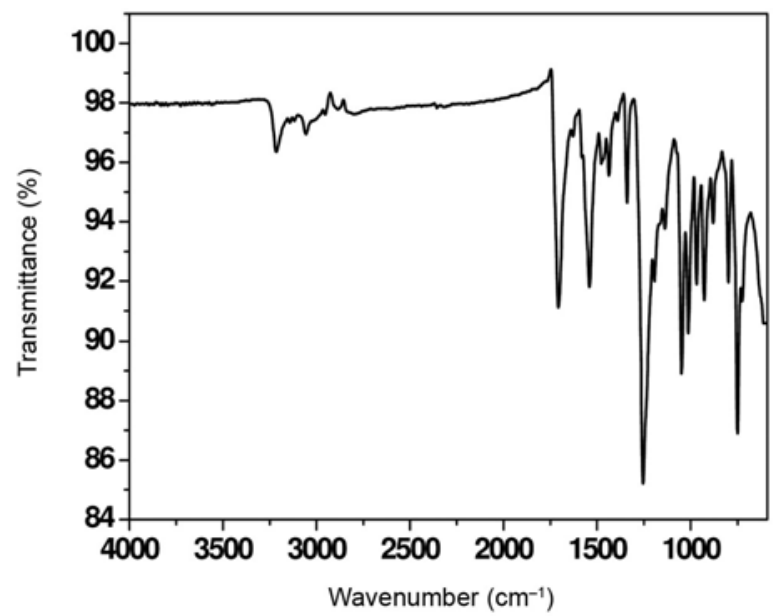

Figure 2. FTIR spectrum of $\mathrm{C}_{7} \mathrm{H}_{8} \mathrm{~N}_{2} \mathrm{O}_{3}$.

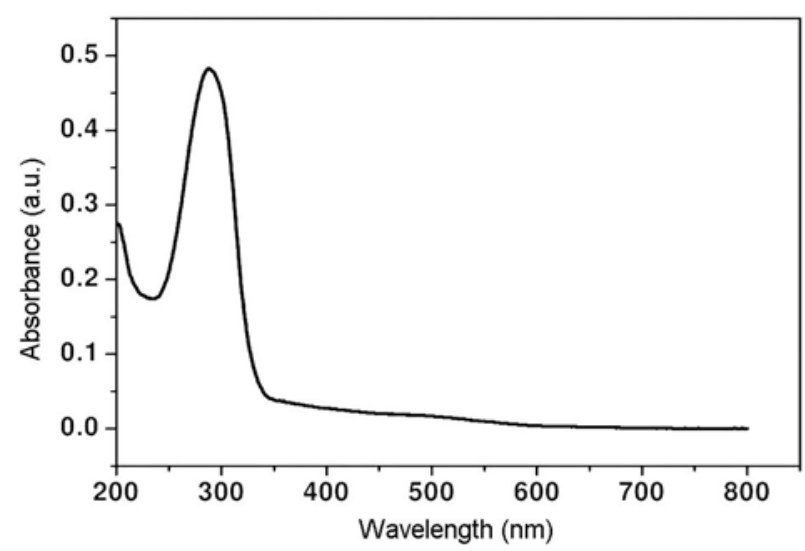

Figure 3. Optical absorption spectrum of MFMC crystal.

absorption peak at $288 \mathrm{~nm}$ (near-UV region) arising from $\pi$ to $\pi^{*}$ transition of $\mathrm{C}=\mathrm{N}$ chromophore present in the compound $^{12}$ and the UV cutoff wavelength is found to be $315 \mathrm{~nm}$. The bandgap value was found to be $3.9 \mathrm{eV}$ using the relation $E=h c / \lambda$. The crystal is transparent in the visible and infrared regions.

\subsection{Thermal analysis}

The recorded TG-DSC curve of MFMC is shown in figure 4. From the TG curve, it is observed that there is

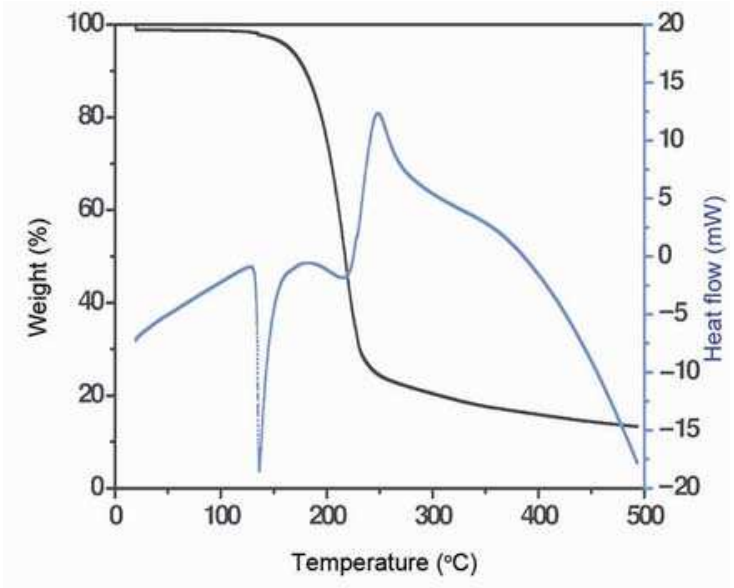

Figure 4. TG-DSC curves of MFMC crystal.

Table 2. Assignments of group frequencies.

\begin{tabular}{ll}
\hline Wavenumber $\left(\mathrm{cm}^{-1}\right)$ & \multicolumn{1}{c}{ Assignment } \\
\hline 3213 & N-H stretching \\
3056 & C-H stretching \\
1707 & C=O normal stretching \\
1628 & C=N stretching \\
1476,1435 & Heterocyclic ring stretching region \\
1390 & CH symmetrical deformation \\
1253 & N=C-H scissoring \\
1137 & C-H in-plane bending \\
1048 & C-N stretching, C-O-C stretching \\
797,749 & C-H out of plane bending \\
724 & N-H wagging \\
\hline
\end{tabular}

only one weight loss. The DSC trace of MFMC shows an endothermic peak at $136.31{ }^{\circ} \mathrm{C}$, which can be attributed to the melting of the material and it is in good agreement with the melting point determined by conventional melting point apparatus. The exothermic peak indicates the material decomposition at $247.91{ }^{\circ} \mathrm{C}$. There is no phase change till the melting, which is suggestive of the purity of the title compound and the absence of lattice water molecules.

\subsection{Dielectric studies}

A sample of size $8.03 \times 4.02 \times 4.08 \mathrm{~mm}^{3}$ was used for the dielectric studies. The dielectric constant and the dielectric loss of MFMC crystal were studied in the frequency 
range from $50 \mathrm{~Hz}$ to $5 \mathrm{MHz}$ at two different temperatures. The variation of dielectric constant with frequency at 50 and $100^{\circ} \mathrm{C}$ is shown in figure $5 \mathrm{a}$. The dielectric constant of the material is high at lower frequency, which may be due to the presence of all types of polarization mechanism that depends on frequency and is also decreasing while increasing the frequency and reaches constant value and becomes independent of the applied frequencies. ${ }^{13}$ This can be associated with the inability of dipoles to rotate rapidly leading to a lag between frequency of oscillating dipole and that of applied field. ${ }^{14}$ The low dielectric constant values at $\mathrm{MHz}$ regions are inferred from the inset graph of figure 5a. The low dielectric constant allows a large operating bandwidth modulation $(>10 \mathrm{GHz})$ by giving low RC time and further eliminating the need for poling while maintaining its refractive index. ${ }^{15,16}$ As the dielectric constant at low frequency region is nearly equal to the optical frequencies, it can be utilized in the minimization of the phase mismatch between electrical and optical pulses in high-speed travelling wave devices. ${ }^{16}$

The variation of dielectric loss with log frequency is shown in figure $5 \mathrm{~b}$ and the inset graph represents the dielectric loss plot at $\mathrm{MHz}$ region. The higher dielectric loss of the material at lower frequency indicates the presence of space-charge polarization, and the lower dielectric loss at higher frequency reveals the good homogeneity of the grown crystal. ${ }^{13}$ The low dielectric constant and dielectric loss at higher frequency enable the compound usage as electrical insulator and its applicability in sensors like strain gauge. ${ }^{17}$ The conductivity of the material is plotted against the frequency at two different temperatures and is shown in figure $5 \mathrm{c}$. The conductivity of the material starts at higher frequency and increases at higher temperature, which are attributed to the increase in drift mobility of thermally activated electric charge carriers according to small polaron hopping mechanism. ${ }^{18}$

\subsection{Microhardness studies}

Mechanical strength of the MFMC crystal was evaluated using the indentation technique. Loads ranging from 1 to $25 \mathrm{~g}$ were applied on the (100) face of the crystal with $5 \mathrm{~s}$ as indentation time. The hardness value of MFMC was found to be $13.6,18.9$ and $19.8 \mathrm{~kg} \mathrm{~mm}^{-2}$, respectively, for 1,3 and $5 \mathrm{~g}$ of applied loads. Cracks started to appear on the crystal at the load of about $10 \mathrm{~g}$ and cracked completely at $25 \mathrm{~g}$ of applied load. The variation of hardness value $\left(H_{\mathrm{v}}\right)$ with the applied indentation load $(P)$ was plotted as shown in figure $6 \mathrm{a}$.
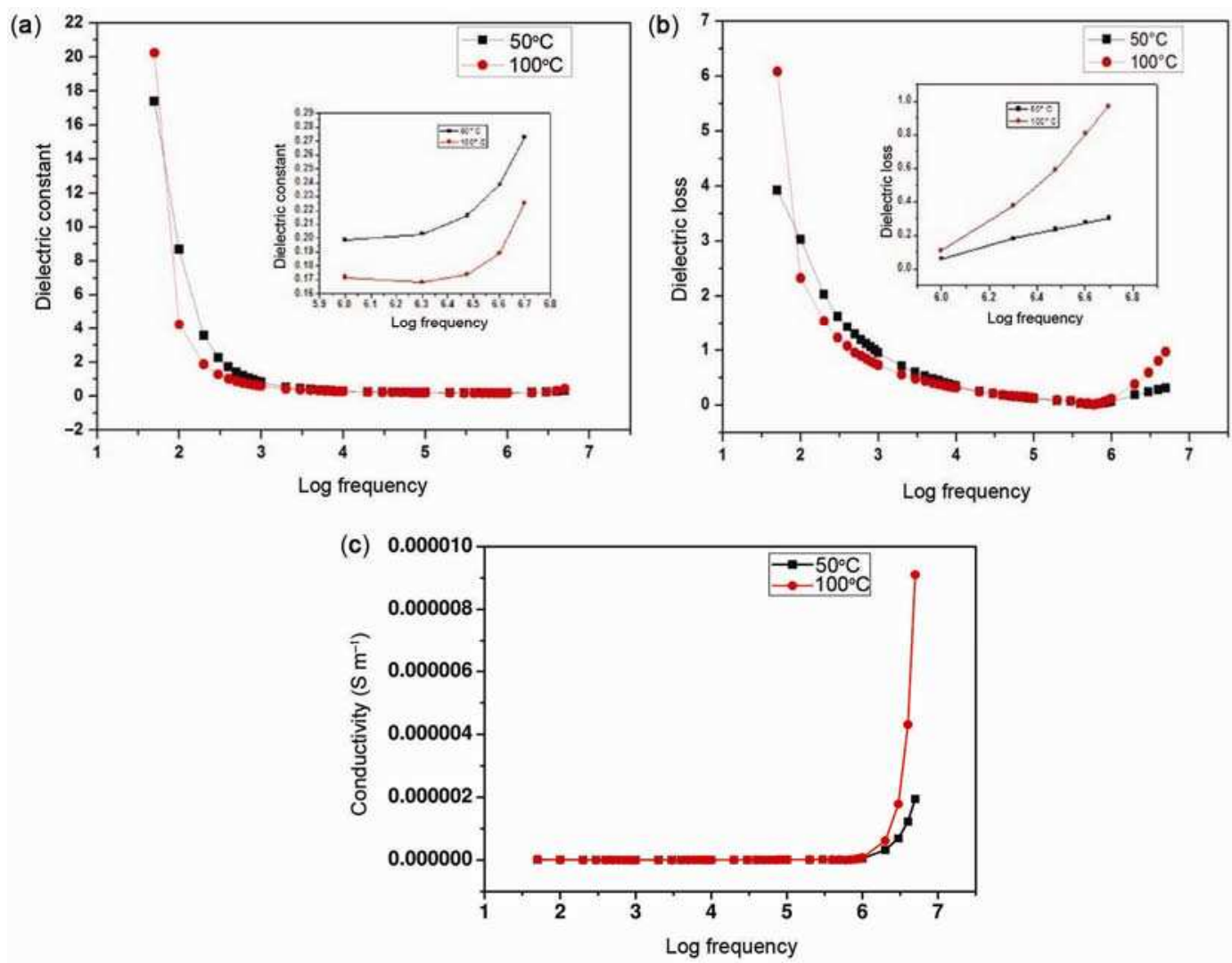

Figure 5. (a) Plot of dielectric constant $v$ s. frequency (inset shows the dielectric constant value at $\mathrm{MHz}$ frequency), (b) plot of dielectric loss vs. frequency (inset shows the dielectric loss value at $\mathrm{MHz}$ frequency) and (c) plot of conductivity $v s$. frequency. 

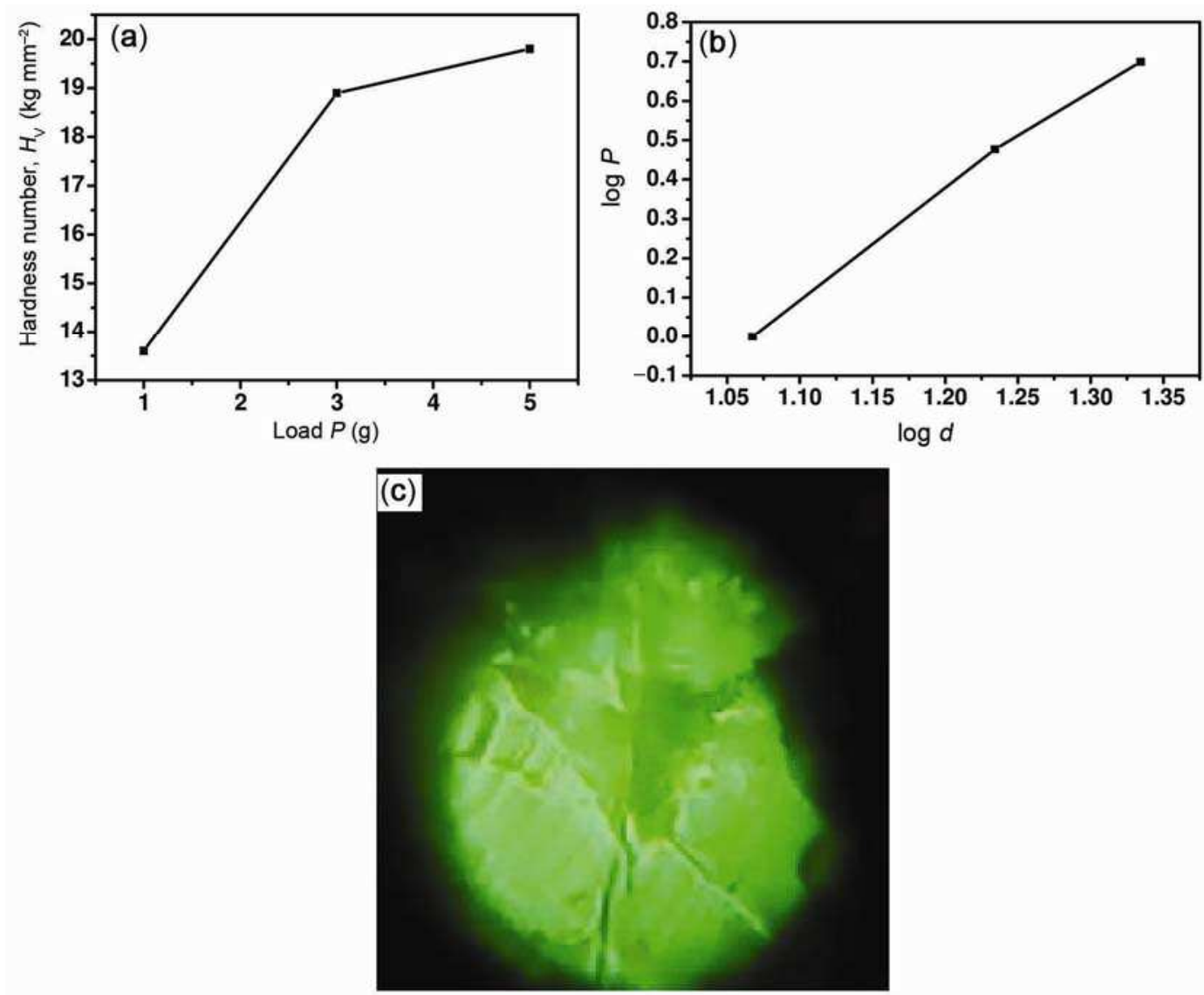

Figure 6. (a) Plot of hardness value vs. applied load, (b) plot between $\log P$ and $\log d$ and (c) micrograph of indentation imprint.

The work hardening co-efficient $(n)$ of the material is found to be 2.8 from the plot of $\log P$ vs. $\log d$ (figure $6 \mathrm{~b}$ ) using Meyer's relation $P=a \cdot d^{n}$, where $P$ is the applied load, $a$ the resistance of the material to initial penetration, $n$ Meyer's index and $d$ the diameter of the indenter. ${ }^{19,20}$ As the value of $n>2$, the material comes under soft material category.

The micrograph of the indentation on the crystal surface (figure 6c) was analysed using optical microscope at a magnification about $400 \times$ in the reflection mode. Radial cracks starting from the corners of the indentation imprints reveal the brittle nature of the crystal. Radial cracks formed as a result of residual tensile stresses are developed during unloading and the cracks were arrested when the stress intensity near the indenter tip equals the material toughness. ${ }^{21}$ Rosette-type deformation is observed near the indentation imprint region. ${ }^{22}$

The first-order stiffness constant can be calculated using Wooster's empirical relation, which is given as $C_{11}=H_{\mathrm{V}}{ }^{7 / 423}$ and the yield strength of the compound can be calculated using the relation $\sigma_{\mathrm{y}}=\left[H_{\mathrm{V}} / 3\right](0.1)^{(n-2)}$, $^{24}$ where $\sigma_{\mathrm{y}}$ is the yield strength, $H_{\mathrm{V}}$ the hardness of the compound and $n$ the work hardening coefficient. The values of stiffness constant and yield strength corresponding to the hardness value are presented in table 3. Stiffness constant and the yield strength values increase with the increase of hardness value. Low values of yield strength prove the low mechanical strength of the title compound.

\subsection{Etching studies}

The (100) face of the MFMC crystal is etched using ethanol as etchant. Figure 7 a shows the surface of the crystal before etching. It can be seen that the rectangular etch pits appeared after etching for $5 \mathrm{~s}$, as shown in figure $7 \mathrm{~b}$. Figure $7 \mathrm{c}$ reveals the rectangular terraced etch pits when etched for $15 \mathrm{~s}$ and the terraced etch pit grows further, while the etching time was increased for $30 \mathrm{~s}$ (figure 7d). It is clear that the grown crystal exhibits rectangular terraced etch pits. ${ }^{25}$

\subsection{SHG efficiency}

The SHG efficiency of the title compound is screened using the Kurtz-Perry powder technique. ${ }^{9}$ The powdered MFMC, the reference KDP and urea samples were sieved with particle size ranging between 45 and $120 \mu \mathrm{m}$. The 

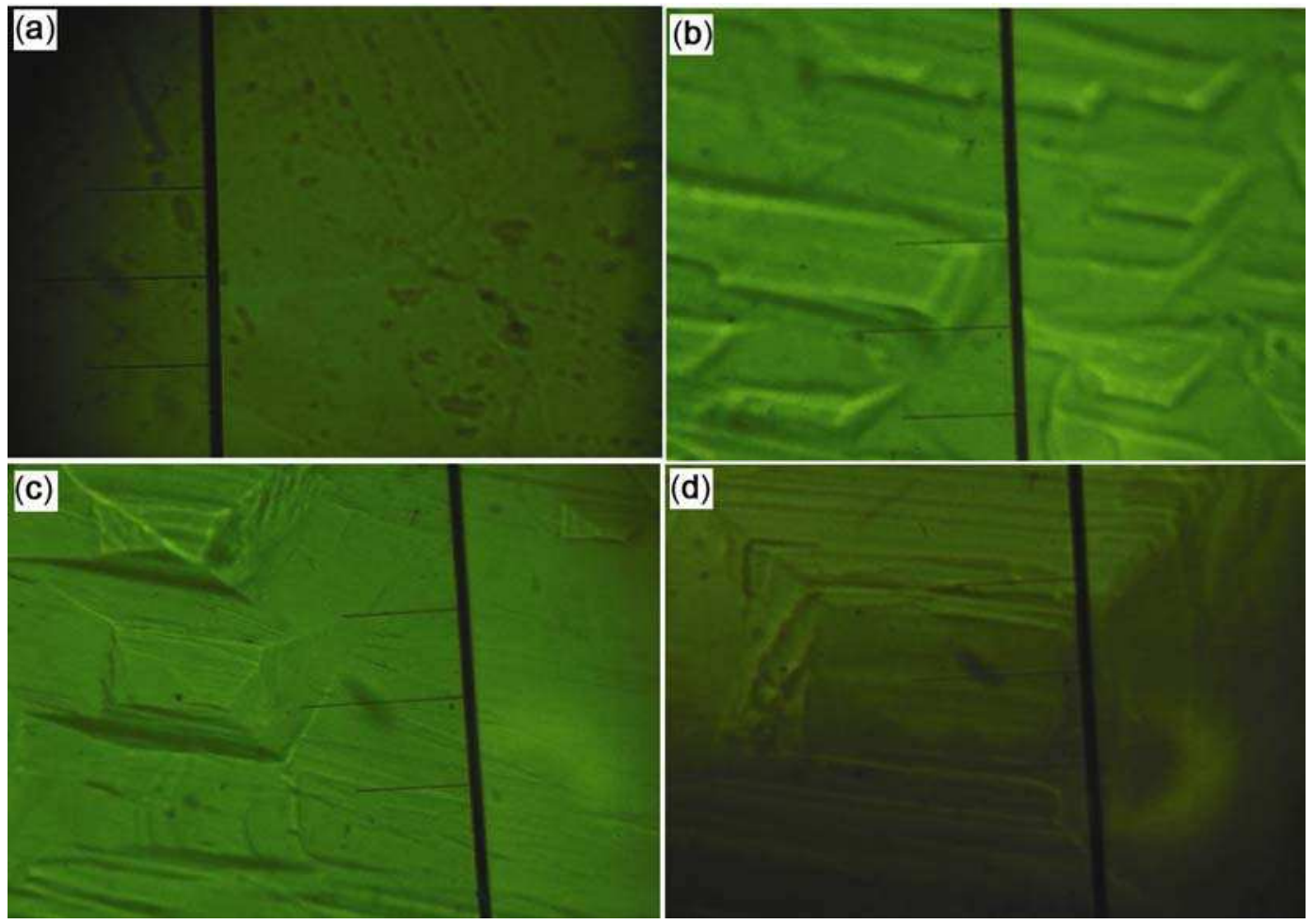

Figure 7. (a) Before etching, (b) etched surface after $5 \mathrm{~s}$, (c) after $15 \mathrm{~s}$ and (d) after $30 \mathrm{~s}$.

Table 3. Calculated stiffness constant and yield strength for the hardness value.

\begin{tabular}{lcc}
\hline $\begin{array}{l}\text { Hardness value, } \\
H_{\mathrm{V}}\left(\mathrm{kg} \mathrm{mm}^{-2}\right)\end{array}$ & $\begin{array}{c}\text { Stiffness constant, } \\
C_{11} \times 10^{14} \mathrm{~Pa}\end{array}$ & $\begin{array}{c}\text { Yield strength, } \\
\sigma_{\mathrm{y}}(\mathrm{MPa})\end{array}$ \\
\hline 13.6 & 0.96 & 0.72 \\
18.9 & 1.71 & 0.99 \\
19.8 & 1.85 & 1.04 \\
\hline
\end{tabular}

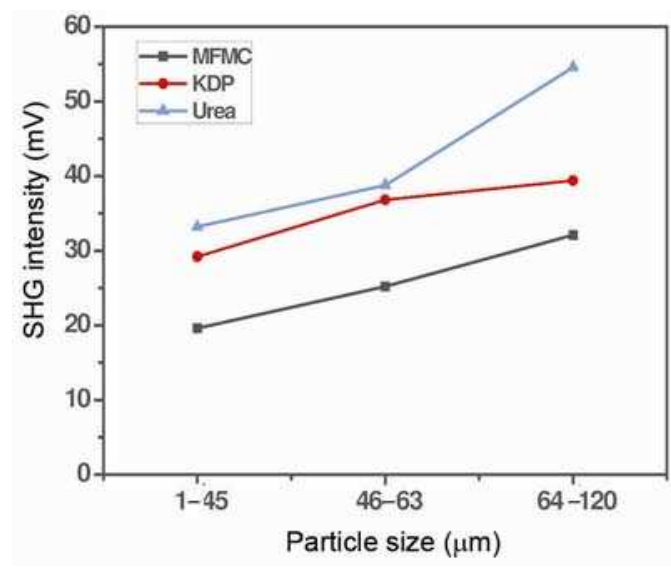

Figure 8. Plot of SHG intensity variation on different particle sizes.

SHG intensity of the main sample along with the reference KDP and urea samples are plotted against particle sizes. At the particle size of about $120 \mu \mathrm{m}$, the SHG efficiency of the title compound is found to be 0.8 and 0.5 times as that of the powdered KDP and urea, respectively. From figure 8, it is obvious that the SHG efficiency of the compound increases with the increasing particle sizes, which confirms Type-I Phase matching. ${ }^{26}$

\section{Conclusions}

An organic MFMC single crystal was grown by using the slow evaporation solution growth technique. Singlecrystal XRD analysis deduced the lattice parameter and confirmed that MFMC crystallizes in monoclinic system. The presence of the functional groups was identified using FTIR spectroscopy analysis. From the UV-DRS studies, the optical absorption of the material was found to be in UV region and was transparent throughout the visible region, and hence makes the crystal suitable for NLO applications in the visible region. The optical bandgap energy value was calculated. The suitability of the grown crystal for optical applications well below its melting point $\left(136^{\circ} \mathrm{C}\right)$ was observed from the TG-DSC trace. The homogeneity and conductivity of the crystal were inferred from the dielectric studies. From the mechanical hardness of the crystal, it was found that the crystal comes under soft material category and brittle in nature with low mechanical stability. Etching studies revealed rectangular terraced etch pits of the grown crystal. The SHG intensity of the compound was measured and 
compared with the standard KDP and urea. It was also confirmed that the compound comes under Type-I phase matching.

\section{Acknowledgements}

G Gomathi acknowledges the Centre for Research, Anna University, Chennai 600 025, for providing Anna Centenary Research Fellowship (Proceeding no. CR/ACRF/ JAN.2011/33). We also acknowledge Prof D Narayana Rao, Department of Physics, University of Hyderabad, for extending his lab facility for SHG studies.

\section{References}

1. Chemla D S and Zyss J 1987 Nonlinear optical properties of organic molecules and crystals (UK: Academic Press) Vol. 1

2. Dalton L R et al 2007 Tutorials in complex photonic media (eds) M W Mccall, G Dewar and N I Zheludev (Bellingam: SPIE Press)

3. Pelusi M D et al 2008 IEEE J. Sel. Top. Quant. Electron. 14529

4. Bosshard Ch et al 1995 Organic nonlinear optical materials (Switzerland: Gordon and Breach Science Publishers)

5. Jiang H and Kloc C 2013 Mater. Res. Bull. 3828

6. Li Y F 2011 Acta Crystallogr. E67 64

7. Kaminsky W 2005 J. Appl. Crystallogr. 38566

8. Kaminsky W 2007 J. Appl. Crystallogr. 40382

9. Kurtz S K and Perry T T 1968 J. Appl. Phys. 393798
10. Silverstein R M, Webster F X and Kiemle D J 2005 Spectrometric identification of organic compounds (USA: John Wiley \& Sons, Inc.)

11. Stuart B 2004 Infrared spectroscopy: fundamentals and applications (England: John Wiley \& Sons, Ltd.)

12. Rao C N R 1961 Ultra-violet and visible spectroscopy, chemical applications (Great Britain: Butterworth \& Co. Ltd.) p 33

13. Sun G H et al 2011 J. Cryst. Growth 316132

14. Awadhia A, Patel S K and Agrawal S L 2006 Prog. Cryst. Growth Charact. Mater. 5261

15. Chen T et al 2012 J. Cryst. Growth 338157

16. Prasad P N and Williams D J 1991 Introduction to nonlinear optical effects in molecules and polymers (New York: Wiley)

17. Gupta K M and Nishu G 2015 Advanced electrical and electronic materials: processes and applications (ed.) T Ashutosh (New Jersy: John Wiley \& Sons, Inc.)

18. Huiling D and Xi Y 2002 J. Phys. Chem. Solids 632123

19. Meyer E 1908 Z. Ver. deut. Ing. 52645

20. Hoyt S L 1924 Trans. Am. Soc. Steel Treating 6396

21. Kruzic J J and Ritchie R O 2003 J. Am. Ceram. Soc. 86 1433

22. Sangwal K 2009 Cryst. Res. Technol. 441019

23. Wooster W A 1953 Rep. Prog. Phys. 1662

24. Cahoon J R, Broughton W H and Kutzaket A R 1971 Metall. Trans. 21971

25. Sangwal K 1987 In Defects in solids: etching of crystals theory, experiment and application (eds) S Amelinckx and Nihoul (The Netherlands: North-Holland Physics Publishing)

26. Ok K M, Chi E O and Halasyamani P S 2006 Chem. Soc. Rev. 35710 